\title{
Primary extradural meningioma arising from the calvarium
}

\author{
N Ravi, ${ }^{1}$ MD; W X Yi, ${ }^{1} \mathrm{PhD}$; L Yu, ${ }^{1} \mathrm{MD}$; H J Ping, ${ }^{2} \mathrm{MB}$ BS; D Z Hao, ${ }^{3} \mathrm{PhD}$ \\ ${ }^{1}$ Department of Radiology, Xiangya Hospital, Central South University, Changsha, Hunan, China \\ ${ }^{2}$ Department of Radiology, Changsha Eighth Hospital, Changsha, Hunan, China \\ ${ }^{3}$ Department of Pathology, Xiangya Hospital, Central South University, Changsha, Hunan, China
}

Corresponding author: WX Yi (cjr.wangxiaoyi@vip.163.com)

\begin{abstract}
Meningiomas are the most common intracranial tumours. Meningiomas arising at other locations are termed primary extradural meningiomas (EDMs) and are rare. Here we report a case of EDM arising from the calvarium - a primary calvarial meningioma (PCM).
\end{abstract}

S Afr J Rad 2013;17(2):70-71. DOI:10.7196/SAJR.836

\begin{abstract}
A 45-year-old woman presented with a left-sided scalp lump of 3 years' duration. The lump began as a small swelling and gradually increased in size. It was not associated with headache or any other neurological deficits. Her past medical history was unremarkable. On examination, the swelling was seen to be situated in the left frontoparietal aspect of the skull, was approximately $5 \mathrm{~cm} \times 5 \mathrm{~cm} \times 0.7 \mathrm{~cm}$ in size, with well-defined borders and a bony consistency. It was non-mobile, nontender and non-pulsatile. Computed tomography (CT) and magnetic resonance imaging (MRI) of the head (Figs 1 - 4) was performed to further characterise the lesion. The patient then underwent a wide resection of the mass, followed by cranioplasty using a titanium mesh. The histopathological diagnosis was a WHO grade I meningioma.
\end{abstract}

\section{Discussion}

Primary extradural meningiomas (EDMs) can arise in any tissue without direct extension from an intracranial tumour. Primary EDMs have a reported incidence of less than $2 \%$ of all meningiomas, and have been reported to arise from scalp tissue, the orbit, paranasal sinuses, nasopharynx, neck, skin, lung, mediastinum, adrenal and paraspinal regions, and fingers. ${ }^{[1]}$ Previously, these tumours were called extracranial, ectopic, extradural, extraneuraxial, cutaneous, calvarial or intraosseous meningiomas. ${ }^{[1-3]}$ However, it has been suggested that the appropriate nomenclature should be primary EDM, to avoid confusion. ${ }^{[1]}$ Primary EDMs have a slight female preponderance $(1: 1.4)^{[2-4]}$ with a bimodal age distribution: The first peak is in the 2nd decade and the next in the 5th 7th decades. ${ }^{[1]}$ Primary EDMs have been classified as extracalvarial (Type I), purely calvarial (Type II) and calvarial with extracalvarial extension (Type III). Types II and III have been further sub-classified into skull convexity (C) and skull base (B) tumours, depending on their location. ${ }^{[1]}$
The most common presenting feature of primary calvarial meningioma $(\mathrm{PCM})$ is a painless, gradually expanding mass lesion. ${ }^{[2,3]}$ On CT examination, the part of the skull that is involved usually has a hyperdense osteoblastic appearance and demonstrates intense contrast enhancement. ${ }^{[1-5]}$ The most common MRI appearance is a hypo-intense $\mathrm{T} 1$ and hyperintense T2 bone lesion, which shows intense homogenous contrast enhancement following IV gadolinium administration. ${ }^{[1-5]}$ The dura underlying the tumour might show contrast enhancement; this is thought to be due to either dural irritation by the tumour or to dural invasion by tumour cells.

Recent studies have suggested that the management strategy for PCM should be based on the histological grade. ${ }^{[2]}$ The treatment strategy for World Health Organization (WHO) grade I (benign) PCMs is complete excision with wide margins. ${ }^{[2]}$ WHO grade II (atypical) and grade III (malignant) tumours are treated with wide excision, followed by adjuvant radiotherapy and/or chemotherapy ${ }^{[2]}$

\footnotetext{
1. Lang FF, Macdonald OK, Fuller GN, DeMonte F. Primary extradural meningiomas: A report on nine cases and review of literature from the era of computerized tomography scanning. J Neurosurg 2000;93(6):940-950. [http://dx.doi.org/10.3171/jns.2000.93.6.0940]

2. Mattox A, Hughes B, Oleson J, Reardon D, McLendon R, Adamson C. Treatment recommendations for primary extradural meningiomas. Cancer 2011;117(1):24-38. [http://dx.doi. org/10.1002/cncr.25384]

3. Elder JB, Atkinson R, Zee CS, Chen TC. Primary intraosseous meningioma. Chin Med J (Engl) 2013;126(7):1390-1391

4. Al-Khawaja D, Murali R, Sindler P. Primary calvarial meningioma. J Clin Neurosci 2007;14(12):1235-1239. [http://dx.doi.org/10.1016/j.jocn.2006.08.010]

5. McGuire TP, Palme CE, Perez-Ordonez B, Gilbert RW, Sándor GK. Primary intraosseous meningioma of the calvaria: Analysis of the literature and case report. Oral Surg Oral Med Oral Pathol Oral Radiol Endod 2007;104:e34-e41.
} 


\section{PICTORIAL INTERLUDE}

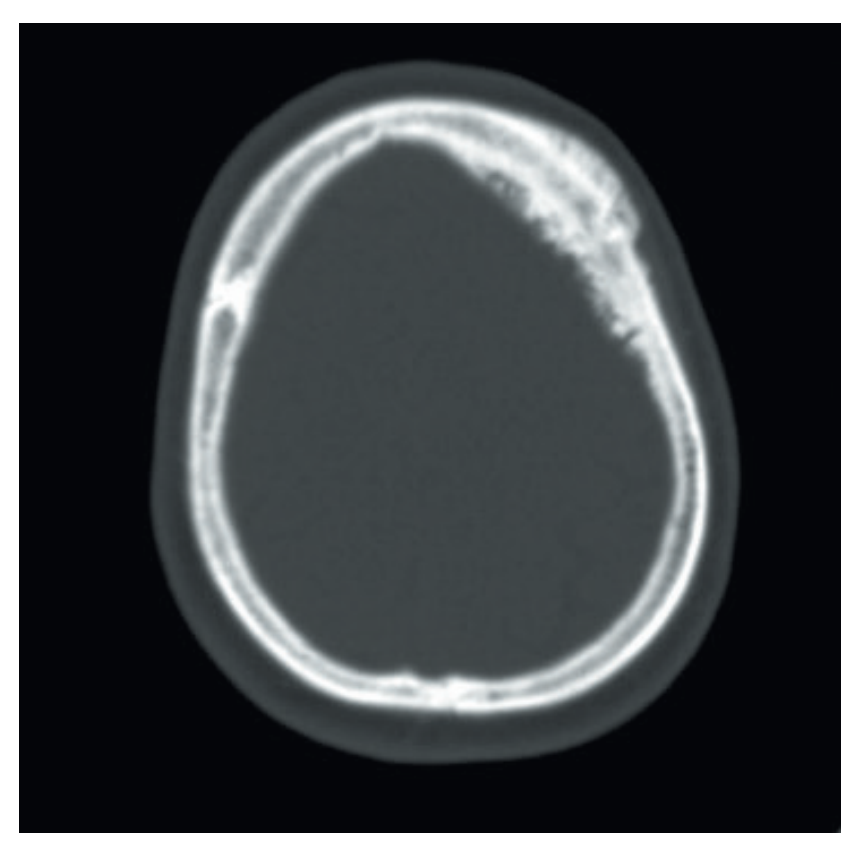

Fig. 1. Axial non-contrast CT image of $2 \mathrm{~mm}$ thickness in bone window settings demonstrates a focal expansile hyperosteotic bony lesion on the left fronto-parietal portion of the skull. The lesion is seen along the course of the coronal suture on the left. There are no evident intracranial abnormalities.

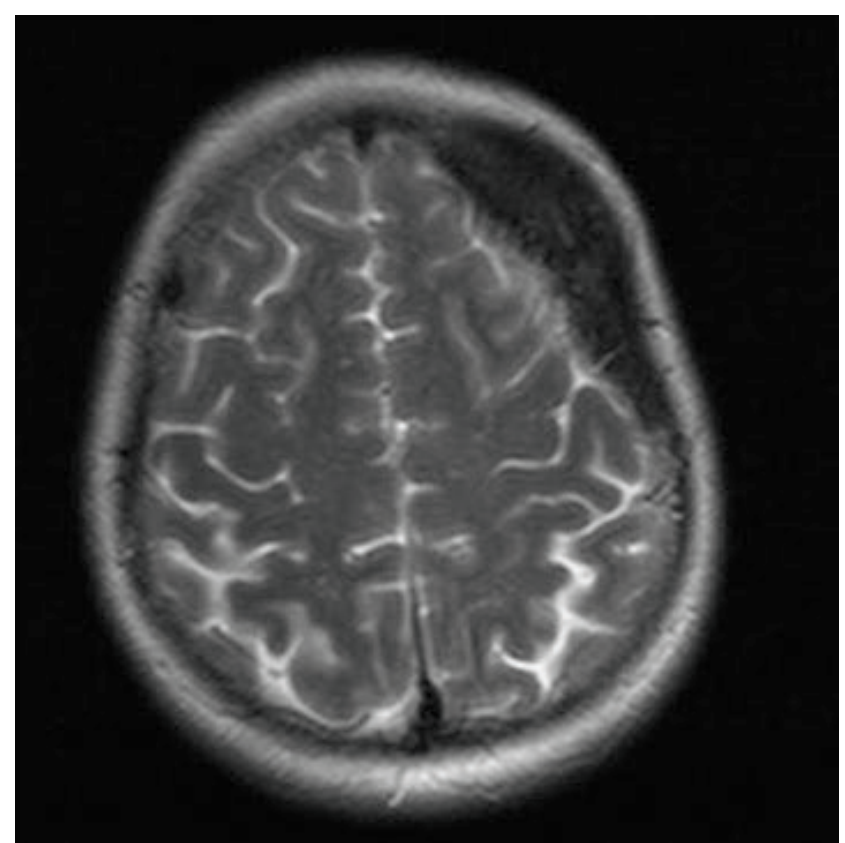

Fig. 3. Axial T2-weighted MR image demonstrates a focal expansile hypointense bone lesion in the superior left frontoparietal region of the skull.

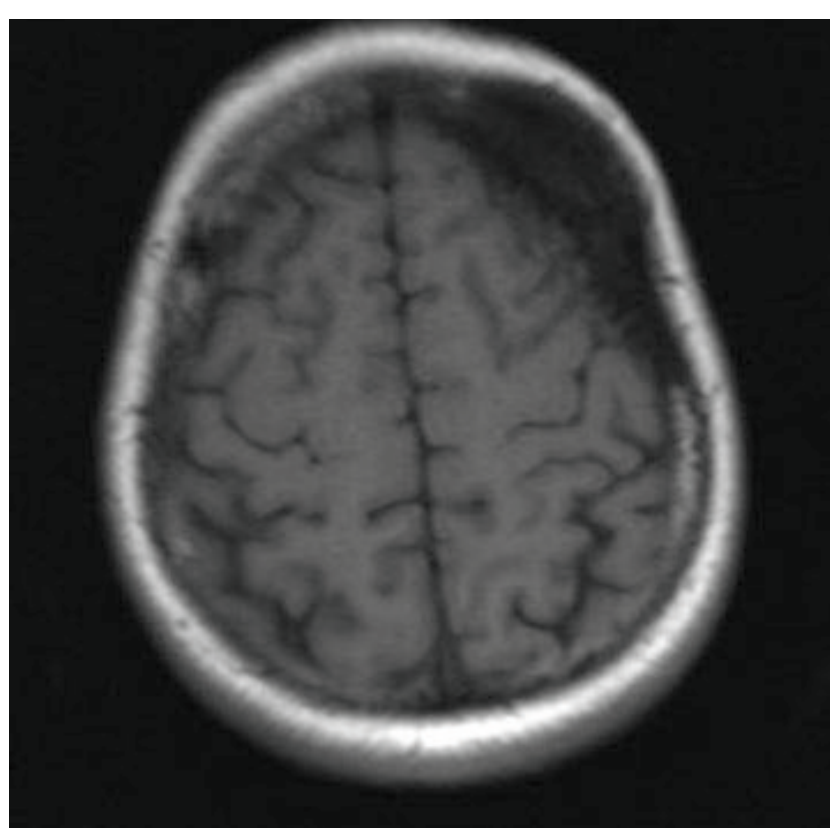

Fig. 2. Axial T1-weighted MR image demonstrates a focal expansile hypointense bone lesion in the superior left fronto-parietal region of the skull. There are no radiologically evident intracranial abnormalities.

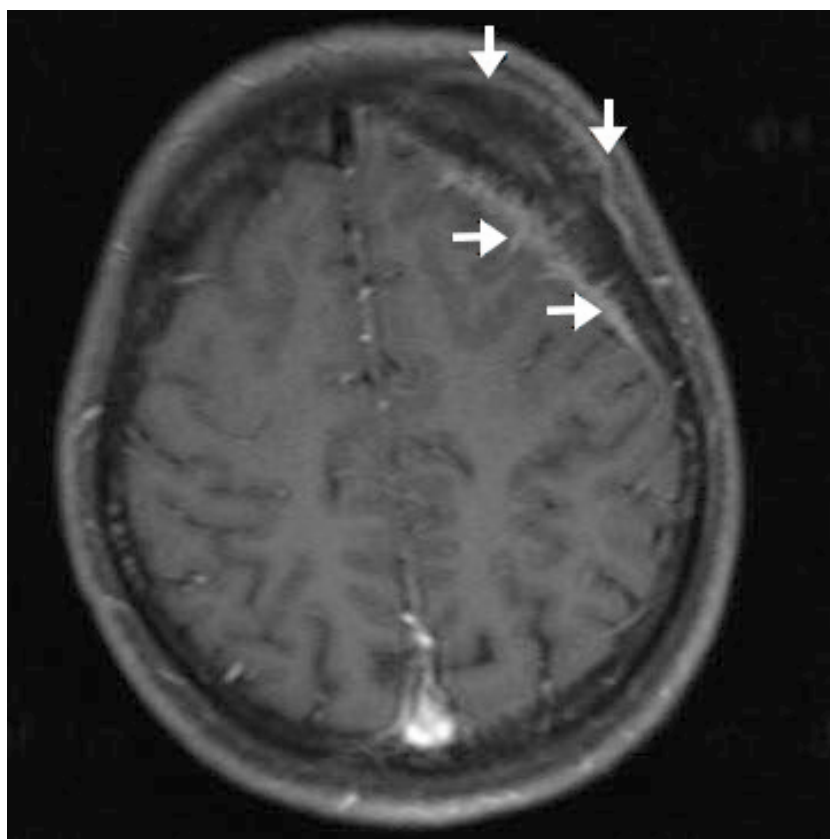

Fig. 4. Axial T1-weighted MR image post gadolinium contrast administration shows a bony lesion measuring approximately $7 \mathrm{~cm} \times 7.2 \mathrm{~cm} \times 2 \mathrm{~cm}$ in size with minimal homogenous contrast enhancement. However, the underlying dura demonstrates an intense contrast enhancement. 